\title{
A Dual Band Antenna Based on a Quarter Mode Substrate Integrated Waveguide
}

\author{
Marc Alvarez, Christos Kalialakis, Fermin Mira \\ Microwave Systems and Nanotechnology Department \\ Centre Tecnològic de Telecomunicacions de Catalunya \\ (CTTC/CERCA) \\ 08860 Castelldefels, Spain \\ christos.kalialakis@cttc.es
}

\author{
Sotirios K. Goudos \\ Radiocommunications Laboratory \\ Department of Physics \\ Aristotle University of Thessaloniki \\ Thessaloniki, Greece \\ sgoudo@physics.auth.gr
}

\begin{abstract}
In this work, a new dual band antenna is presented. The antenna is implemented on a quarter mode Substrate Integrated Waveguide (SIW) technology by introducing a slit shaped modification. The dual band antenna concept has been demonstrated for the $2.4 \mathrm{GHz}$ and $5 \mathrm{GHz} \mathrm{WiFi}$ bands but the approach is readily applicable to centimeter and millimeter wave applications due to the low loss characteristics of SIW. Good agreement has been observed between simulations and measurements on a prototype.
\end{abstract}

Keywords- Substrate Integrated Waveguides, Antennas, Dual Band Antennas, Cavity Antennas

\section{INTRODUCTION}

The developments of wireless communications are driving the research for printed antennas that offer low cost and lightweight solutions [1]. Microstrips and variants (such as Coplanar Waveguides, CPW) have dominated the field of printed microwave antennas [2]. With the advent of new systems such as $5 \mathrm{G}$ and mobile satellite communications frequencies of operation are pushed towards centimetric and millimeter wave frequencies [3]. In these frequencies, waveguides have been traditionally used because of low loss and high quality factors. A promising technology that combines the benefits of printed and waveguide technology is the Substrate Integrated Waveguide (SIW). SIW technology has already produced during the last decade a considerable number of antennas and circuits, both passive and active [4]. However, SIW in its basic form suffers from size and bandwidth limitations. Reduced size has been achieved by taking advantage of field symmetry in the waveguide; half [5], quarter [6] and eighth moded SIW structures [7] have been proposed.

Considering the multitude of applications and bands, a multiband antenna operation is highly desirable $[8,9]$. This work is focused on the dual band operation. Dual band operation can be achieved by properly modifying the shape of a resonant antenna. A half mode SIW dual band antenna has been recently suggested in [10]. Going further down in size, a dual band antenna based on Quarter Mode SIW(QMSIW) is proposed here using the single band resonator introduced in [6].
In Section II, the antenna design process is described in detail. In Section III, results on a prototype working on $2.4 \mathrm{GHz}$ and $5 \mathrm{GHz}$ is presented. Section $\mathrm{V}$ is devoted to conclusions.

\section{ANTENNA DESIGN}

\section{A. Quarter Mode SIW resonators}

Consider a substrate integrated waveguide implemented on a substrate of dielectric constant $\varepsilon_{\mathrm{r}}$ and height h. The SIW is a dielectric filled waveguide with the lateral walls replaced by a series of cylindrical metallized vias. Waveguide equivalence $[11,12]$ provides the way to determine the dimensions of the SIW by studying the corresponding dielectric filled metal waveguide.

The quarter mode SIW is generated by starting from a full SIW cavity (Fig.1). The cavity walls are realized by two parallel series of cylindrical vias. By taking advantage of the symmetry a half mode cavity can be made and subsequently a quarter mode(QM). In order, to generate the necessary field symmetries, an excitation through the diagonal is needed.
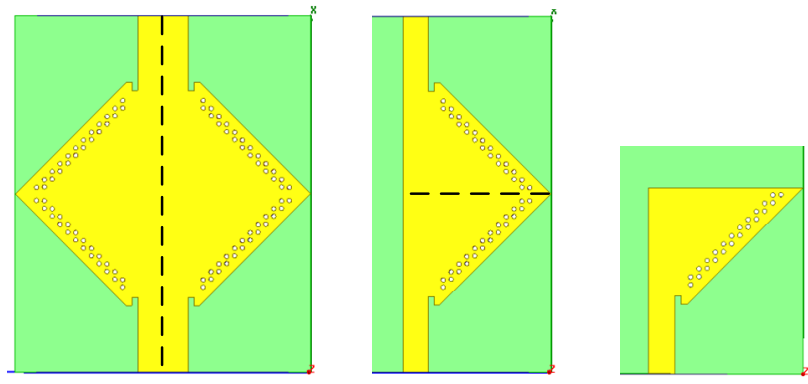

Fig. 1. Conceptual evolution of a SIW resonator to the Half and Quarter Mode Resonator.

The QM waveguide dimensions with reference to Fig.2 are [9]

$$
L_{Q M S I W}^{\text {eff }}=W_{Q M S I W}^{\text {eff }}=\frac{W_{R E S}^{\text {eff }}}{2}+\Delta W
$$


where

$\Delta W=h\left(0.05+\frac{0.30}{\varepsilon_{r}}\right) x \ln \left(0.79 \frac{W_{R E S}^{e f f}}{4 h^{3}}+\frac{52 W_{R E S}^{e f f}-261}{h^{2}}+\frac{38}{h}+2.77\right)$

The frequency of each of the cavity modes of the QM waveguide is given by

$f_{Q M S I W}^{m n p}=\frac{c_{0}}{2 \pi \sqrt{\mu_{r} \varepsilon_{r}}} \sqrt{\left(\frac{m \pi}{2 L_{Q M S I W}^{e f f}}\right)^{2}+\left(\frac{n \pi}{h}\right)^{2}+\left(\frac{p \pi}{2 W_{Q M S I W}^{e f f}}\right)^{2}}$

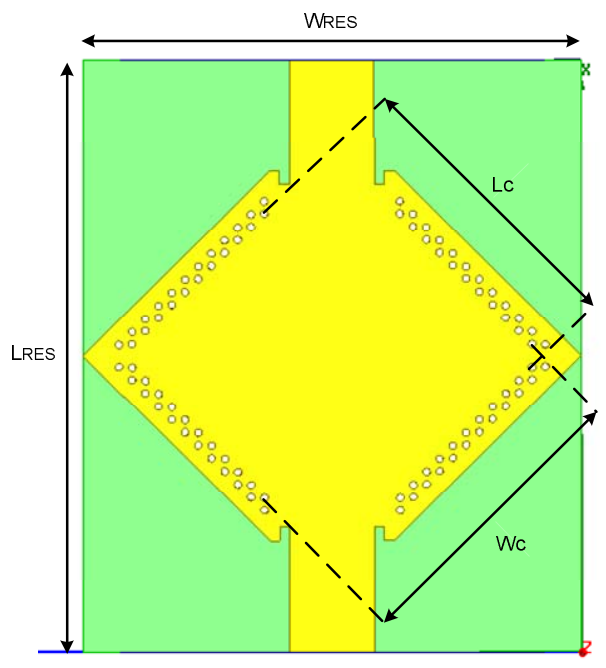

(a)

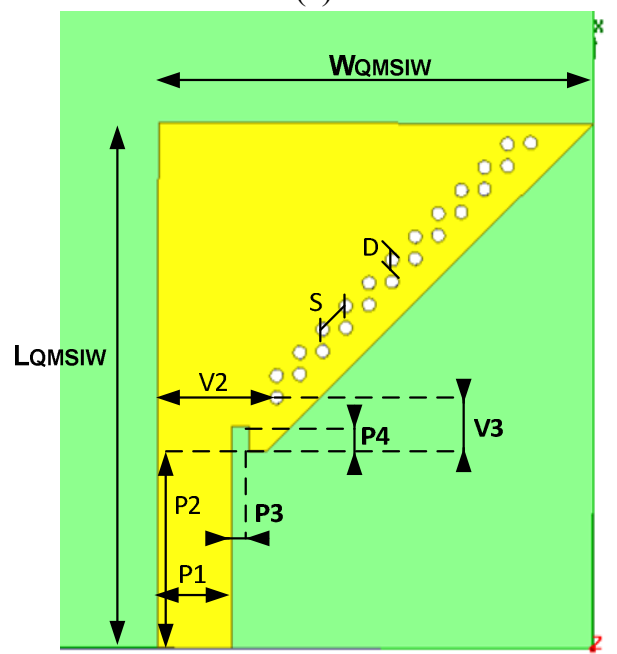

(b)

Fig. 2. (a) Design variables related to the SIW cavity. (b) Design variables for the QM SIW resonator.
For the antenna in this work, the first band is provided by the first resonant mode with a corresponding frequency $f_{Q M S I W}^{101}$ calculated from (3) for $\mathrm{m}=1, \mathrm{n}=0, \mathrm{p}=1$. The dimensions for the $2.4 \mathrm{GHz}$ mode are given in Table I with reference to Fig.2. A Rogers RT5580 substrate is used throughout as the substrate $(\varepsilon \mathrm{r}=2.2, \mathrm{~h}=1.57 \mathrm{~mm})$. The dielectric loss tangent is 0.0011. The established EMCAD Ansys HFSS [13] is used throughout for the full wave simulations.

TABLE I

DESIGN VARIABLES FOR THE FIRST MODE TE 101 AT $2.4 \mathrm{GHZ}$

\begin{tabular}{|l|l|l|}
\hline No & \multicolumn{1}{|c|}{ Variable } & Value(mm) \\
\hline 1 & $\mathrm{~L}_{\mathrm{RES}}$ & 48 \\
\hline 2 & $\mathrm{~W}_{\mathrm{RES}}$ & 40 \\
\hline 3 & $\mathrm{~L}_{\mathrm{C}}$ & 22.63 \\
\hline 4 & $\mathrm{~W}_{\mathrm{C}}$ & 22.63 \\
\hline 5 & $\mathrm{~S}$ & 1.5 \\
\hline 6 & $\mathrm{D}$ & 0.6 \\
\hline 7 & $\mathrm{~V}_{1}$ & 2.45 \\
\hline 8 & $\mathrm{~V}_{2}$ & 5.45 \\
\hline 9 & $\mathrm{P}_{1}$ & 3.4 \\
\hline 10 & $\mathrm{P}_{2}$ & 9 \\
\hline 11 & $\mathrm{P}_{3}$ & 0.8 \\
\hline 12 & $\mathrm{P}_{4}$ & 1.1 \\
\hline 13 & $\mathrm{~W}_{\mathrm{QMSIW}}$ & 44 \\
\hline 14 & $\mathrm{~L}_{\mathrm{QMSIW}}$ & 44 \\
\hline
\end{tabular}

\section{B. Dual Band Design Using a Slit}

The contribution of this work is the introduction of a second resonant frequency. The second resonance is engineered by introducing a slit governed by three design parameters, length width and relative position as shown in Fig.3. The initial dimensions are calculated by considering an equivalent cavity with an extra length introduced by the slit. The dimensions are optimized using HFSS

For the matching, a stub loaded structure is adopted which is also bent to reduce the overall dimensions. The circuit has been first designed in Keysight's ADS software [14] (Fig.4) using two open (labeled Stub oc1, oc2 in Fig.3) and two circuit stubs (labeled Stub cc1, cc2 in Fig.3). The ADS matching circuit dimensions were then fed back to HFSS for an antenna-circuit EM co-optimization (Fig.3). The simulated matching values achieved were better than $-15 \mathrm{~dB}$ at $2.4 \mathrm{GHz}$ and better than $-29 \mathrm{~dB}$ at $5 \mathrm{GHz}$. The final values of the variables in Fig. 3 are given in Table II.

The choice of matching circuit in a microstrip form is not restrictive. In fact, other matching structures could be utilized in case of centimetric and millimetric waves to avoid the microstrip feeding network radiation loss at these frequencies. 


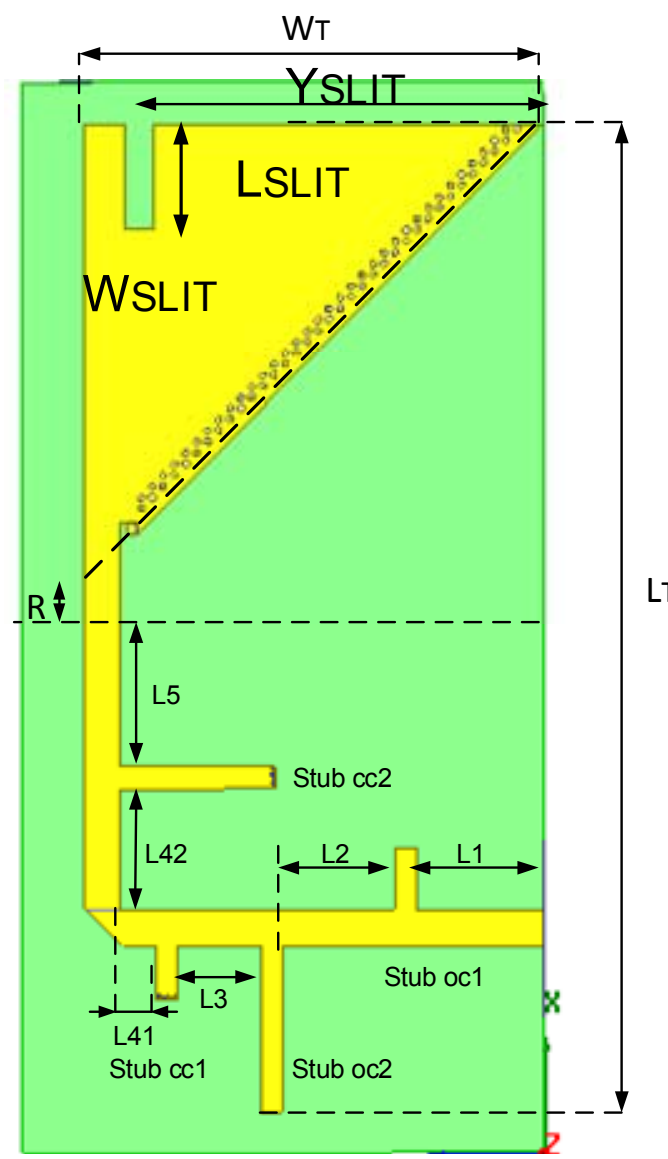

Fig. 3. Design variables for the dual band Antena QMSIW including a general stub based matching circuit.

TABLE II. DESIGN VARIABLES FOR THE DUAL BAND ANTENANS INCLUDING MATCHING CIRCUIT

\begin{tabular}{|l|l|l|}
\hline No & Variable & \multicolumn{1}{|c|}{ Value(mm) } \\
\hline 1. & $\mathrm{~L} 1$ & 12.2 \\
\hline 2. & $\mathrm{~L} 2$ & 10.9 \\
\hline 3. & $\mathrm{~L} 3$ & 8 \\
\hline 4. & $\mathrm{~L} 41$ & 3.4 \\
\hline 5. & $\mathrm{~L} 42$ & 11.47 \\
\hline 6. & $\mathrm{~L} 5$ & 3.2 \\
\hline 7. & $\mathrm{~W}_{\mathrm{T}}$ & 44 \\
\hline 8. & $\mathrm{~L}_{\mathrm{T}}$ & 97.4 \\
\hline 9. & $\mathrm{~W}_{\mathrm{SLIT}}$ & 2.7 \\
\hline 10. & $\mathrm{~L}_{\mathrm{SLIT}}$ & 10 \\
\hline 11. & $\mathrm{Y}_{\mathrm{SLIT}}$ & 37.3 \\
\hline 12. & $\mathrm{~W}_{\mathrm{OC} 1}$ & 2 \\
\hline 13. & $\mathrm{~W}_{\mathrm{OC} 2}$ & 2.1 \\
\hline 14. & $\mathrm{~W}_{\mathrm{CC} 1}$ & 2 \\
\hline 15. & $\mathrm{~W}_{\mathrm{CC} 2}$ & 2.1 \\
\hline 16. & $\mathrm{~L}_{\mathrm{OC} 1}$ & 6 \\
\hline 17. & $\mathrm{~L}_{\mathrm{OC} 2}$ & 15.8 \\
\hline 18. & $\mathrm{~L}_{\mathrm{CC} 1}$ & 5.1 \\
\hline 19. & $\mathrm{~L}_{\mathrm{CC} 2}$ & 14.8 \\
\hline
\end{tabular}

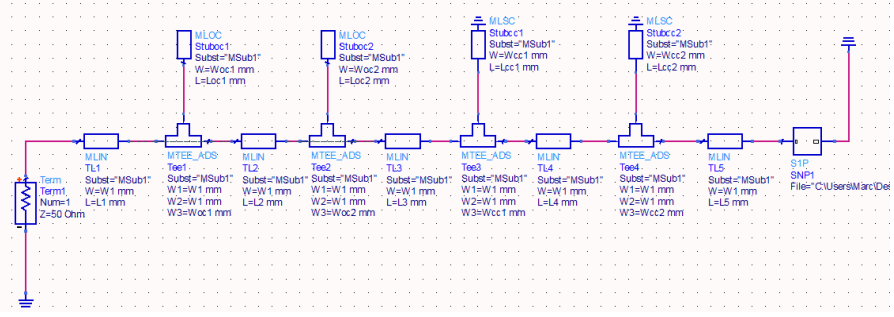

Fig. 4. The matching circuit for matching $2.4 \mathrm{GHz}$ and $5 \mathrm{GHz}$ in ADS corresponding to the layout in Fig.3 .

\section{ANTENNA MEASUREMENTS}

In this section, results are presented for a prototype that operates in $2.4 \mathrm{GHz}$ and $5 \mathrm{GHz}$ bands (Fig 5). For the vias metallization, silver epoxy paste was utilized that was cured in an oven.

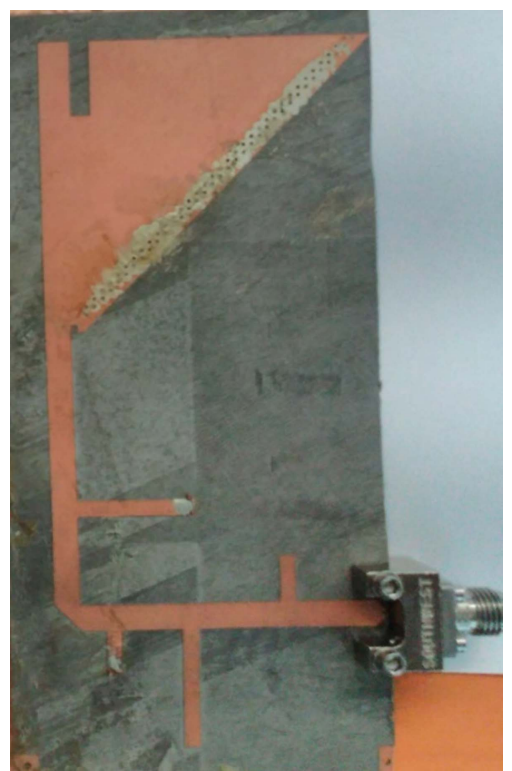

Fig. 5. Prototype dual band antenna that operates in $2.4 \mathrm{GHz}$ and $5 \mathrm{GHz}$.

The operation of the antenna in two bands can be seen in Fig.6 where the return loss (both simulated and measured) is shown. The experimental return loss of the first resonance is weak whereas the second resonance agrees well with the simulation. The weak first resonance is attributed to the laser milling used. The heating involved caused significant warping of the soft substrate which was found to affect the depth and position of the resonance. Placing the prototype on a rigid metallic surface stabilized the resonance but the warping was still considerable at the area of the matching stub. It is expected that this problem will be solved in the next prototype. 


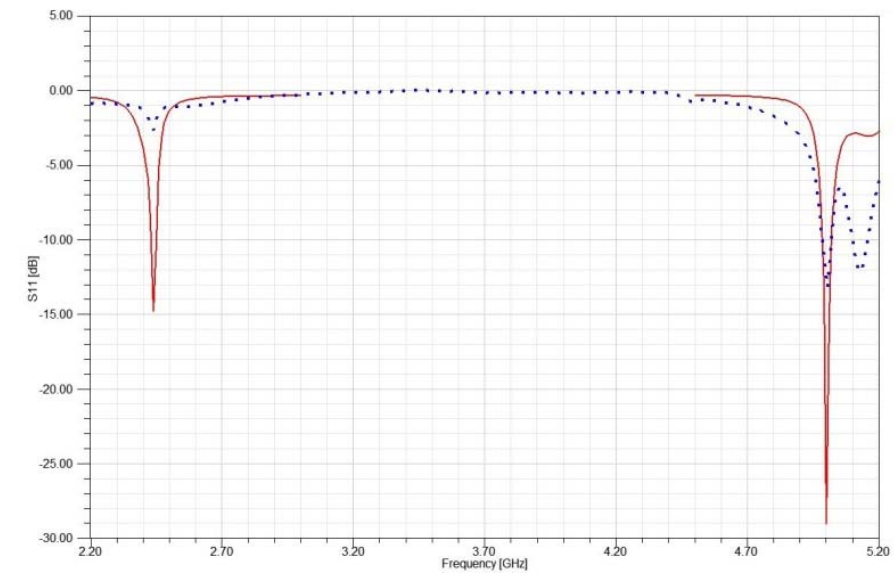

Fig. 6. Measured (dotted lines) and simulated(continuous) return loss of the antenna prototype

The radiation patterns at the two frequencies were measured in an ETS-Lindgren anechoic chamber. The simulation results are also shown in Fig. 7 for $2.4 \mathrm{GHz}$ and Fig. 8 for $5 \mathrm{GHz}$. Good agreement is observed. The substrate warping is a possible reason for the difference in Fig. 8.

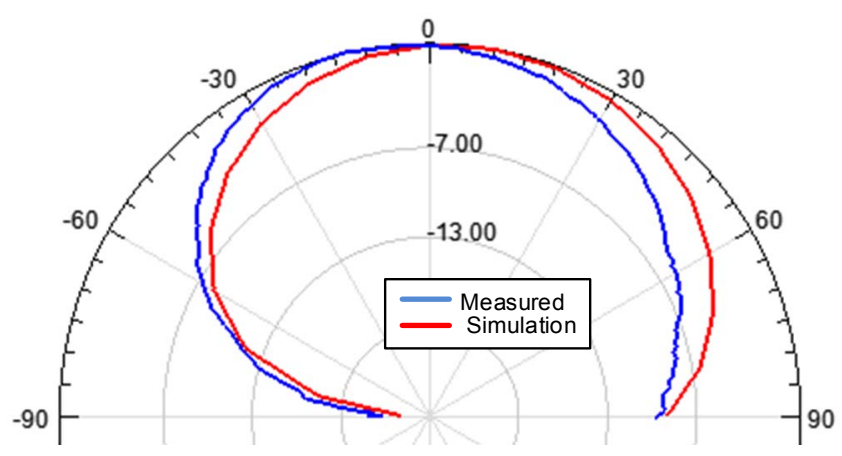

Fig. 7. Radiation pattern of the QMSIW dual band antenna at $2.4 \mathrm{GHz}$

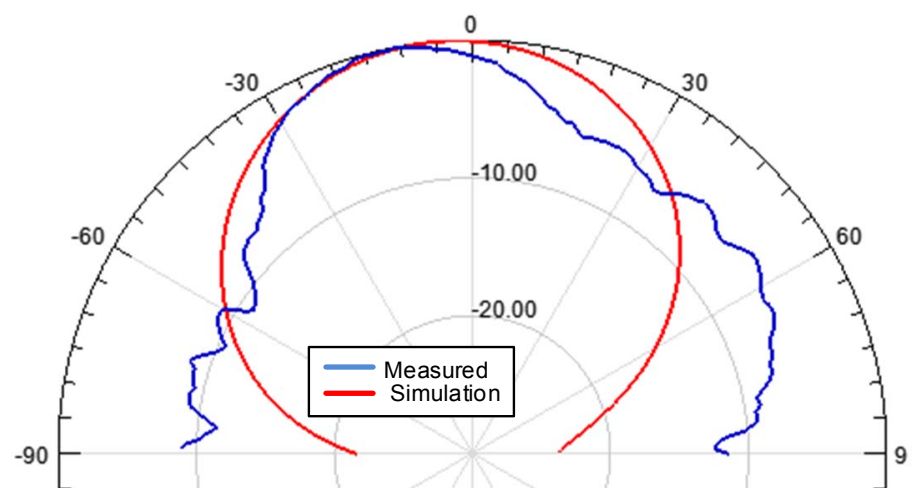

\section{CONCLUSION}

A new dual band antenna is implemented in quarter mode SIW technology. The second band is achieved by introducing a slit and presents a further reduction in size compared to existing SIW antennas. There is good agreement between simulation and measurements for a prototype operating at 2.4 and $5 \mathrm{GHz}$. Future work will focus on centimeter and millimeter wave prototypes.

\section{ACKNOWLEDGMENTS}

The work of Christos Kalialakis has been supported by the European Union Horizon 2020 research and innovation programme under the Marie Skłodowska-Curie grant agreement No 654734. The work of M.Alvarez and F.Mira has been supported by the Catalan Government under Grant 2014 SGR 1551.

\section{REFERENCES}

[1] R.Waterhouse, ed. Printed antennas for wireless communications. New York: John Wiley \& Sons, 2007.

[2] R.Garg,. Microstrip antenna design handbook. Artech house, 2001.

[3] T. S. Rappaport et al., "Millimeter Wave Mobile Communications for 5G Cellular: It Will Work!, ” IEEE Access, vol. 1, pp. 335-349, 2013.

[4] M. Bozzi, A.Georgiadis, and K.Wu,"Review of substrate-integrated waveguide circuits and antennas," IET Microw. Antennas Propag., vol. 5, pp. 909-920,2011.

[5] Q.Lai,C.Fumeaux, W.Hong, R. Vahldieck,"Characterization of the propagation properties of the half-mode substrate integrated waveguide", IEEE Trans. Microw. Theory Tech., vol.57, pp. 19962004,2009

[6] C.Jin, R.Li, A.Alphones, X. Bao, "Quarter-Mode Substrate Integrated Waveguide and its Application to antennas design," IEEE transactions on antennas and propagation, vol. 61,pp. 2921-2928, June 2013.

[7] M. Mujumdar, and A. Alphones,"Eighth-mode Substrate Integrated Resonator Antenna at $2.4 \mathrm{GHz}$," IEEE Antennas and Wireless Propagation Letters, pp. 853-856, 2016

[8] X.Wang, W. Chen, Z.Feng, "Multiband antenna with parasitic branches for laptop applications", Electronics letters, vol. 43, pp.1012-103, 2007

[9] T. H.Kim, D. C. Park, "Compact dual-band antenna with double L-slits for WLAN operations", IEEE Antennas and Wireless Propagation Letters, 2005, pp.249-252.

10] S.Banerjee, B.Rana, and S.K. Parui, "SIW based compact and dual-band equilateral triangular antennas", Journal of Electromagnetic Waves and Applications, 2016.

[11] F. Xu, K.Wu, "Guided-wave and leakage characteristics of substrate integrated waveguide", IEEE Trans. Microw. Theory Tech., vol.53, pp. 66-73, 2005.

12] D.Deslandes, K.Wu, "Accurate modeling, wave mechanisms, and design considerations of a substrate integrated waveguide", IEEE Trans. Microw. Theory Tech., vol.54, pp. 2516-2526, 2006.

[13] Ansys HFSS, Electromagnetics Suite[computer software], (Release 16.2).

[14] Keysight Advanced Design System (ADS) [computer software]. (Version http://www.keysight.com/find/eesof-technicalreference

Fig. 8. Radiation pattern of the QMSIW dual band antenna at $5 \mathrm{GHz}$ 